\title{
Development of a Korean Version of Pro-Change's Processes of Change Measure for Effective Stress Management
}

\author{
Ke Deng1, Akira Tsuda ${ }^{2}$, Satoshi Horiuchi' ${ }^{3}$, Euiyeon Kim ${ }^{4}$, Terumi Matsuda5, \\ Satomi Doi 6 , Aki Tsuchiyagaito', Hisanori Kobayashi ${ }^{3}$, Kwangshik Hong7 \\ ${ }^{1}$ Institute of Comparative Studies of International Cultures and Societies, Kurume University, Kurume, Japan \\ ${ }^{2}$ Department of Psychology, Kurume University, Kurume, Japan \\ ${ }^{3}$ University of Rhode Island, Kingston, USA \\ ${ }^{4}$ Department of Education, Inha University, Inchon, Korea \\ ${ }^{5}$ Graduate School of Psychology, Kurume University, Kurume, Japan \\ ${ }^{6}$ Graduate School of Psychological Science, Health Sciences University of Hokkaido, Tobetsu, Japan \\ ${ }^{7}$ Department of Elemenrary Education, Jeonju National University of Education, Jeonju, Korea \\ Email: sztouka@yahoo.co.jp
}

Received July 2014

\section{Abstract}

This study aimed to develop a Korean version of Pro-change's processes of change measure for effective stress management (PPSM). Effective stress management refers to any form of healthy activity practiced at least 20 min per day to manage stress. PPSM includes 30 items and consists of two higher-order and ten first-order factors. It measures the covert and overt strategies that individuals use to initiate and maintain effective stress management, namely the processes of change. The participants included 542 Korean college students. The Korean version of PPSM was found to consist of two higher-order and ten first-order factors, which was consistent with the original PPSM. Reliability was examined in terms of internal consistency. Cronbach's alpha coefficients ranged from 0.41 to 0.83 , which was largely compatible to those of the original, Japanese, and Chinese PPSM. The Korean PPSM version was validated against the stages of change for effective stress management. The differences in the scores of the processes of change were largely consistent with those of previous studies.

\section{Keywords}

Processes of Change, Stages of Change, Effective Stress Management, Transtheoretical Model

\section{Introduction}

Managing stress - that is, coping effectively with stressful situations-is a public concern in East Asian countries such as Japan and South Korea [8]. Stressful experiences have been found to be linked to numerous chronic 
diseases such as cardiovascular diseases [10]. To prevent stress-related health problems, it is important to practice effective stress management [6], which refers to any form of healthy activity practiced for at least 20 min per day to reduce a person's perceived stress [11] (Pro-Change Behavior Systems, Inc., 2003). Unfortunately, it is estimated that more than half of Korean college students do not engage in effective stress management [4], which points to a need for some assistance in practicing effective stress management. To design such interventions, appropriate behavioral change theories need to be identified.

One such model is the transtheoretical model (TTM) of behavioral change [12]. This model describes behavioral change through five stages: precontemplation (having no intention to begin in the next six months), contemplation (intending to begin in the next six months), preparation (intending to begin in the next month), action (having been practicing for less than six months), and maintenance (having been practicing for at least six months). Other factors such as processes of change are assumed to facilitate stage transitions. Processes of change refer to the covert and overt strategies that individuals use to progress to the next stage. Ten processes of change are assumed to be needed to facilitate a stage transition. Table 1 gives a definition for each of these processes, which are divided into higher-order processes, and include both experiential and behavioral processes.

Use of processes of change has been found to depend on stages of change. Pertinent to this study, Horiuchi et al. [6] reported tight relationships between stages and processes of change for effective stress management. The least use of these processes was found in the precontemplation stage, whereas experiential processes were most frequently used in the preparation stage, and behavioral processes were most frequently used in the preparation, action, and maintenance stages. These results suggest that increasing and maintaining these processes are important to progress through the stages.

Model-based interventions have been found to successfully increase the proportion of participants practicing effective stress management [3] [5]. The development of a model-based intervention could provide Korean researchers and practitioners with a new intervention approach to help college students initiate effective stress management practices. To do this, however, we first need a measure that can assess how frequently each individual uses each process of change. Such a measure not only enables the identification of specific processes that affect stage progression but also allows practitioners to assess the interventions based on the TTM. Unfortunately, no measure is currently available for assessing the processes of change for effective stress management.

Pro-change's processes of change measure for effective stress management (PPSM) [2] have been shown to be a reliable and valid measure. PPSM includes 30 items, with two higher-order factors (experiential and behavioral processes) and ten first-order factors (individual processes). Its structure was evaluated and found to be acceptable, with a comparative fit index (CFI) of 0.76. Reliability was also found to be acceptable with Cronbach $\alpha$ coefficients ranging from 0.59 to 0.79 . Validity was confirmed with significant inter-stage differences in the use of the processes. This study aimed to develop a Korean version of PPSM.

Table 1. Processes of change for effective stress management.

\begin{tabular}{|c|c|}
\hline Processes of change & Definition \\
\hline \multicolumn{2}{|l|}{ Experiential processes } \\
\hline Consciousness raising & Increasing awareness \\
\hline Dramatic relief & Reacting emotionally to warnings about stress \\
\hline Environmental re-evaluation & Considering how the practice or lack of stress management affects others \\
\hline Self-re-evaluation & Realizing that stress management can enhance one’s identity \\
\hline Social liberation & Acknowledging how society is changing to encourage stress management \\
\hline \multicolumn{2}{|l|}{ Behavioral processes } \\
\hline Self-liberation & Making a commitment to initiate stress management \\
\hline Helping relationships & Listing and utilizing support for stress management \\
\hline Reinforcement management & Using positive reinforcement and reward for stress management \\
\hline Counter conditioning & Substituting new and positive behavioral choices \\
\hline Stimulus control & Restructuring one's environment for stress management \\
\hline
\end{tabular}




\section{Method}

\subsection{Participants and Procedures}

The institutional review board of Kurume University approved this study. Five-hundred and eighty Korean college students gave informed written consent for the participation, and completed the questionnaire packet. The data of 22 students were removed as they had not been stressed over the past month, which was assessed using the staging algorithm described below. A further 16 students were removed because of missing data. The final data of 542 Korean college students were subject to the analyses. Of the 542 students, 206 were male and 336 were female. The average age was 20.77, with a standard deviation of 2.91 years. The participants included $35.98 \%$ freshmen $(n=195), 37.27 \%$ sophomores $(n=202), 22.64 \%$ juniors $(n=123)$, and $4.06 \%$ seniors $(n=$ 22).

\subsection{Measures}

\subsubsection{Processes of Change}

PPSM (Evers et al., 2000) [2] was translated into Korean for this study, with permission from Pro-Change Behavior Systems, Inc. First, two Korean psychologists translated the PPSM English items into Korean. Another two psychologists checked that the original meaning of each item had not changed through the translation. Each participant was asked to rate each of the 30 PPSM items on a five-point Likert scale ( $1=$ Never to $5=$ Repeatedly).

\subsubsection{Stages of Change}

The participants' stages of change were assessed using the Korean version of Pro-Change's staging algorithm [7]. The participants were asked whether they effectively managed stress in their daily life. They chose from one of the six items that represented the five stages and a further item "I had no stress." A one-week test-retest reliability was found to be acceptable. Validity was confirmed by demonstrating that the relationships of depressive symptoms were consistent with the predictions [7].

\subsection{Statistical Analyses}

The analyses were conducted using SPSS 20 for Windows and R. The statistical significance was set as $\mathrm{p}<$ 0.05 .

First, to examine if the Korean PPSM version consisted of two higher-order and ten first-order factors, a confirmatory factor analysis was conducted. Three items were expected to represent one first-order factor and five first-order factors were expected to represent one higher-order factor. The root mean square error of approximation (RMSEA) and CFI were used to evaluate the model fit. RMSEA values of less than 0.08 show an acceptable fit, and those of 0.76 or larger show a compatible fit to the original measure. Then, reliability was evaluated in terms of internal consistency. The Cronbach $\alpha$ coefficient was calculated for each subscale. Finally, to validate the Korean PPSM version, we examined whether the differences in mean values for the processes of change for effective stress management across the five stages were consistent with those of Horiuchi et al. [6]. Tenone-way analyses of variance (ANOVAs) were conducted. The model assumes that the processes of change cause stage transitions [13], so they were put in the ANOVAs as independent variables. However, this study aimed to examine the relationships between the stages and processes of change. In previous cross-sectional studies of the relationships between processes and stages of change for effective stress management [1] [2] [6], processes of change were put into the analyses as a dependent variable. Therefore, this study also put the processes of change as the dependent variable.

\section{Results}

\subsection{Factor Structure}

A confirmatory factor analysis was conducted to examine whether the original PPSM factor structure was acceptable in this sample. The two higher-order and ten first-order factor models showed an acceptable fit to the data $(\mathrm{CFI}=0.81$; RMSEA $=0.07$ ). The factor loadings ranged from 0.32 to 0.89 (Table 2). Strong correlations emerged between the first- and higher-order factors with the standardized path coefficients ranging from 0.60 to 1.01 (Figure 1). 
Table 2. Item factor loadingsfor the Korean $\mathrm{PPSM}^{*}$ version.

\begin{tabular}{|c|c|}
\hline Item & Factor loadings \\
\hline \multicolumn{2}{|l|}{ Consciousness raising $(\alpha=0.75)$} \\
\hline I search for information about how to deal with stress in a healthy way. & 0.73 \\
\hline I think about information on healthy stress management. & 0.83 \\
\hline I read about people who have successfully used healthy stress management techniques. & 0.72 \\
\hline \multicolumn{2}{|l|}{ Dramatic relief $(\alpha=0.53)$} \\
\hline I get upset when people ignore health warnings about not practicing stress management. & 0.68 \\
\hline It upsets me to hear about people who do not manage their stress in a healthy way. & 0.72 \\
\hline I react emotionally to warnings about stress. & 0.42 \\
\hline \multicolumn{2}{|l|}{ Environmental re-evaluation $(\alpha=0.73)$} \\
\hline I think about how managing my stress in a healthy way would have a positive effect on the people around me. & 0.66 \\
\hline I stop to think about how my stress can affect others. & 0.74 \\
\hline I consider how managing my stress would benefit my family and friends. & 0.77 \\
\hline \multicolumn{2}{|l|}{ Self-re-evaluation $(\alpha=0.70)$} \\
\hline I feel good about myself when I use healthy strategies to manage my stress. & 0.66 \\
\hline I believe that I am a more confident person when I successfully manage my stress. & 0.56 \\
\hline I see myself as a more responsible person when I manage my stress. & 0.75 \\
\hline \multicolumn{2}{|l|}{ Social liberation $(\alpha=0.46)$} \\
\hline Society is providing more healthy options for managing stress. & 0.32 \\
\hline I notice that managing stress is becoming a greater concern in our society. & 0.55 \\
\hline I notice stress management techniques being discussed more openly. & 0.67 \\
\hline \multicolumn{2}{|l|}{ Self-liberation $(\alpha=0.54)$} \\
\hline I refuse to use unhealthy strategies to manage my stress. & 0.36 \\
\hline I tell myself that I can choose to manage my stress in a healthy way. & 0.65 \\
\hline I promise myself that I will take active steps to manage my stress. & 0.65 \\
\hline \multicolumn{2}{|l|}{ Helping relationships $(\alpha=0.77)$} \\
\hline I have someone I can count on when I experience stress in my life. & 0.66 \\
\hline There is at least one person who can provide feedback about how I'm managing my stress. & 0.78 \\
\hline An important person in my life helps me confront my problems with managing my stress. & 0.89 \\
\hline \multicolumn{2}{|l|}{ Reinforcement management $(\alpha=0.83)$} \\
\hline Friends and family say something positive when I use healthy strategies to manage stress. & 0.77 \\
\hline Others praise my choice to use healthy strategies to manage stress. & 0.84 \\
\hline I am rewarded by others when I use healthy strategies to manage stress. & 0.81 \\
\hline \multicolumn{2}{|l|}{ Counter conditioning $(\alpha=0.41)$} \\
\hline When I start to feel stressed out, I take a short break to relax. & 0.33 \\
\hline I focus on one problem at a time instead of becoming overwhelmed by stress. & 0.39 \\
\hline When I begin to feel stressed, I engage in healthy activities I enjoy. & 0.59 \\
\hline \multicolumn{2}{|l|}{ Stimulus control $(\alpha=0.57)$} \\
\hline I save some healthy pleasures, like taped TV shows, for times of stress. & 0.39 \\
\hline I keep things at home that remind me to use healthy stress management techniques. & 0.64 \\
\hline I keep reminders to think positively when I feel stressed. & 0.64 \\
\hline
\end{tabular}

Note: PPSM = Pro-Change’s processes of change measure for effective stress management. ${ }^{*} \odot$ 2004. Pro-Change Behavior Systems, Inc. All Rights Reserved.

\subsection{Reliability}

The Cronbach's $\alpha$ coefficient was calculated for each subscale (Table 2), and ranged from 0.41 to 0.83. While the $\alpha$ coefficients for the five subscales were at 0.60 or above, those for the other subscales were below 0.59 . 


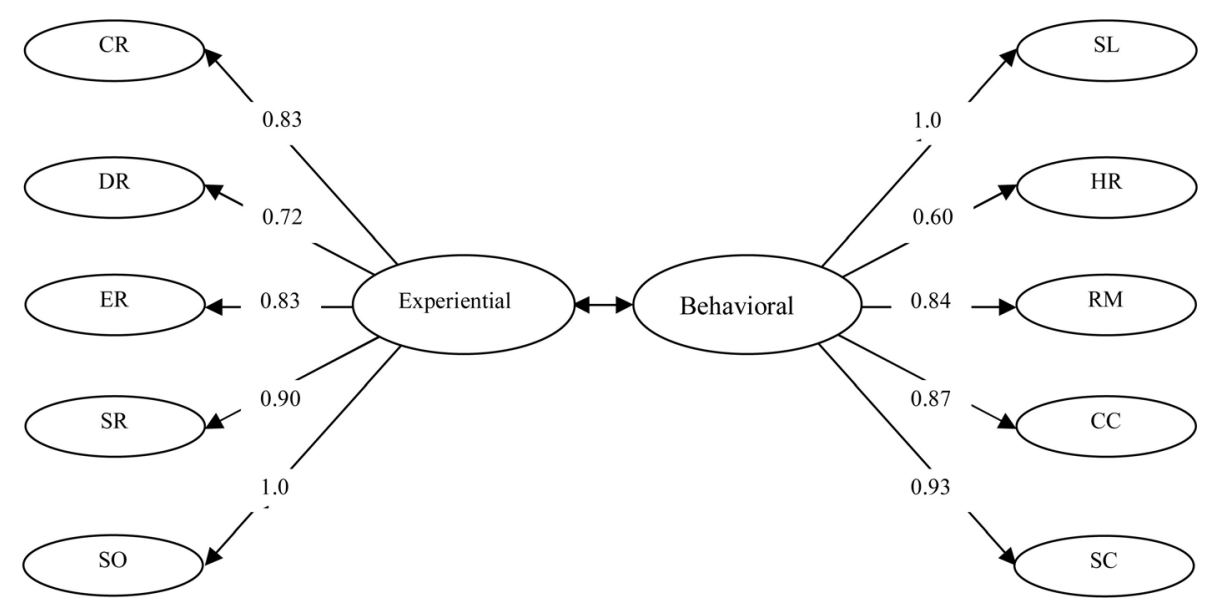

Figure 1. Hierarchal model for the processes of change for effective stress management. $\mathrm{CR}=$ consciousness raising, DR = dramatic relief, ER = environmental re-evaluation, $\mathrm{SR}=$ self-reevaluation, $\mathrm{SO}=$ social liberation, $\mathrm{SL}=$ self-liberation, $\mathrm{HR}$ = helping relationships, $\mathrm{RM}=$ reinforcement management, $\mathrm{CC}=$ counter conditioning, $\mathrm{SC}=$ stimulus control.

\subsection{Validity}

The distribution of the participants was as follows: $16.79 \%$ in precontemplation $(n=91), 21.59 \%$ in contemplation $(\mathrm{n}=117), 26.20 \%$ in preparation $(\mathrm{n}=142), 12.18 \%$ in action $(\mathrm{n}=66)$, and $23.25 \%$ in maintenance $(\mathrm{n}=$ 126). The distribution was independent of gender $\left(\chi(4)^{2}=2.89, \mathrm{p}=0.58\right)$.

To examine the validity of the Korean PPSM version, the relationships between the stages and processes of change were examined using ten ANOVAs. The differences in the processes of change scores across the stages are shown in Figure 2 and Figure 3. The results of these ANOVAs are summarized in Table 3. The ANOVAs found significant main effects in the stages for all ten processes. The differences in the scores for the processes of change showed patterns of increase in the processes' use across the stages.

\section{Discussion}

This is the first study in which a measure for the processes of change for effective stress management was developed in South Korea. After translating PPSM into Korean, its factor structure, reliability, and validity were examined. The Korean PPSM version provides aninitial tool to assess the use of each process of change for effective stress management. The model enables researchers and practitioners to develop interventions that help people manage stress by tailoring the intervention content to an individual's stages. This requires the identification of specific processes that cause forward stage transitions, such as the progression from precontemplation to contemplation. The Korean PPSM version is expected to not only enable researchers identify such processes but also to assist practitionerse valuate the effects of interventions based on the TTM.

The two higher- and ten first-order factor structures in the Korean PPSM version were supported by a confirmatory factor analysis. The value of CFI was compatible to that of the original PPSM ( 0.81 versus 0.76$)$. In addition, the RMSEA value was found to be acceptable. However, note that two of the ten path coefficients between the first- and higher-order factors exceeded 1.00. These abnormal values indicated that ten of the individual processes of change were highly correlated. These high correlations were also found in a sample of Chinese college students, where Deng et al. [1] reported that the path coefficients ranged from 0.60 to 0.93 and ten processes were found to be highly correlated. Because of these high correlations, it could be argued that the validity of the division of the processes of change into ten processes should be re-examined. We agree with this possible argument, but accept the two higher-order and ten-factor model because of the following two reasons. First, five experiential and five behavioral processes are clearly differentiated in the content, and differential techniques are required to facilitate each process. Second, we aim to develop the Korean PPSM version, whose development will enable further explorations into the validity on Korean populations.

The reliability of the Korean PPSM version was examined in terms of internal consistency. The $\alpha$ coefficient for each subscale ranged from 0.41 to 0.83 . The subscales for dramatic relief, social liberation, self-liberation, 


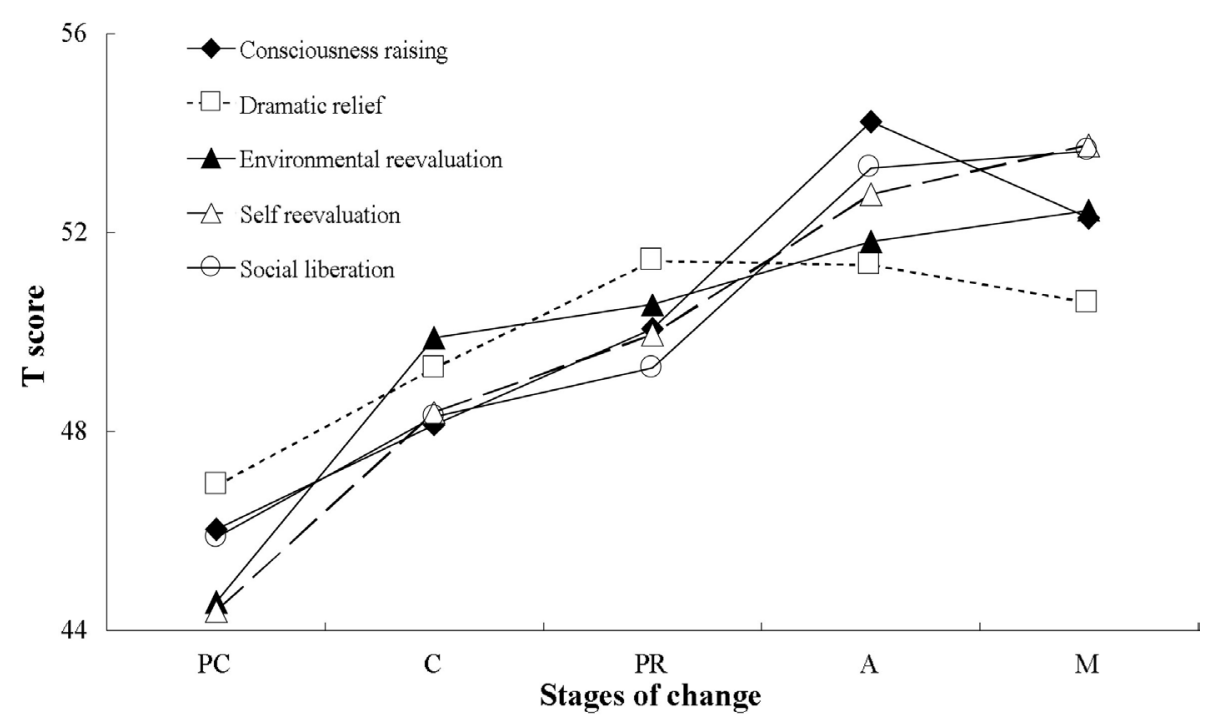

Figure 2. Differences in the scores for the experiential processes across the stages of change for effective stress management. Note: $\mathrm{PC}=$ precontemplation; $\mathrm{C}=$ contemplation; $\mathrm{PR}=$ preparation; $\mathrm{A}=$ action; $\mathrm{M}=$ maintenance.

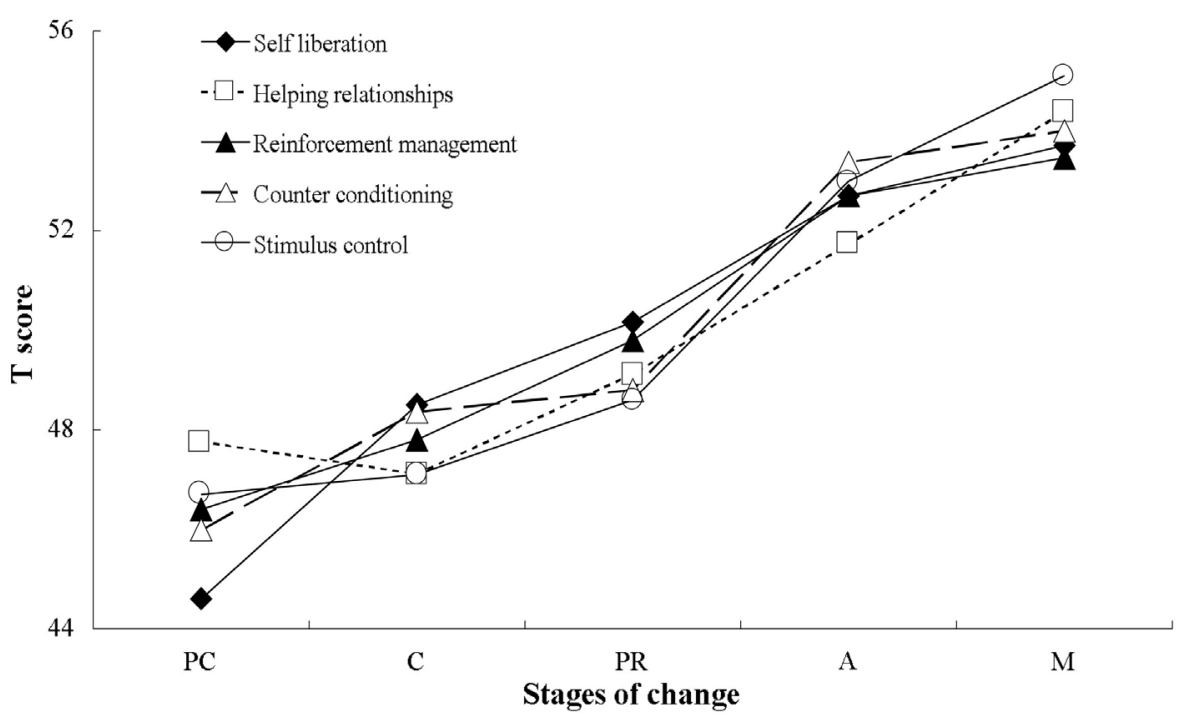

Figure 3. Differences in the behavioral processes scores across the stages of change for effective stress management. Note: $\mathrm{PC}=$ precontemplation; $\mathrm{C}=$ contemplation; $\mathrm{PR}=$ preparation; $\mathrm{A}=$ action; $\mathrm{M}=$ maintenance.

counter conditioning, and stimulus control were below acceptable values (i.e., $>0.60$ ). Among the five subscales, the low value of the counter conditioning subscale $(\alpha=0.41)$ was in line with that of the original PPSM ( $\alpha=$ 0.59) [2]. The three items that assess counter conditioning include three coping options to manage stress. It is expected that the broadness of the subscale resulted in low $\alpha$ values. Relatively low $\alpha$ values for dramatic relief ( $\alpha=0.53)$, self-liberation $(\alpha=0.54)$, and stimulus control $(\alpha=0.57)$ were lower than those for the original PPSM, but were largely consistent with those for the Chinese $(\alpha=0.49-0.65)$ [1] and Japanese PPSM $(\alpha=0.55$ - 0.73) [6]. Note that the low $\alpha$ value for social liberation $(\alpha=0.46)$ was substantially lower than that for the Chinese $(\alpha=0.67)$ [1] and Japanese PPSM ( $\alpha=0.69)$ [6]. In summary, nine of the ten subscales in the Korean PPSM version showed compatible levels of internal consistency to the Japanese and Chinese PPSM. These results suggested that the Korean PPSM version can be useful for cross-cultural studies across mainland China, Japan, and South Korea. 
Table 3. Summary of analyses of variances.

\begin{tabular}{|c|c|c|}
\hline Processes of change & $\mathrm{F}(4,537)$ & Results of Tukey HSD \\
\hline \multicolumn{3}{|l|}{ Experiential processes } \\
\hline Consciousness raising & $9.78^{* *}$ & $\mathrm{PC}<\mathrm{PR}, \mathrm{A}, \mathrm{M} ; \mathrm{C}<\mathrm{A}, \mathrm{M} ; \mathrm{PR}<\mathrm{A}$ \\
\hline Dramatic relief & $3.54 * *$ & $\mathrm{PC}<\mathrm{PR}, \mathrm{A}$ \\
\hline Environmental re-evaluation & $9.84 * *$ & $\mathrm{PC}<\mathrm{C}, \mathrm{PR}, \mathrm{A}, \mathrm{M}$ \\
\hline Self-re-evaluation & $13.45^{* *}$ & $\mathrm{PC}<\mathrm{PR}, \mathrm{A}, \mathrm{M} ; \mathrm{C}<\mathrm{A}, \mathrm{M} ; \mathrm{PR}<\mathrm{M}$ \\
\hline Social liberation & $11.76^{* *}$ & PC, C, PR < A, M \\
\hline \multicolumn{3}{|l|}{ Behavioral processes } \\
\hline Self-liberation & $14.01^{* *}$ & $\mathrm{PC}<\mathrm{C}, \mathrm{PR}, \mathrm{A}, \mathrm{M} ; \mathrm{C}<\mathrm{A}, \mathrm{M} ; \mathrm{PR}<\mathrm{M}$ \\
\hline Helping relationships & $11.20^{* *}$ & $\mathrm{PC}<\mathrm{M} ; \mathrm{C}<\mathrm{A}, \mathrm{M} ; \mathrm{PR}<\mathrm{M}$ \\
\hline Reinforcement management & $9.96 * *$ & $\mathrm{PC}, \mathrm{M}<\mathrm{A}, \mathrm{M} ; \mathrm{PR}<\mathrm{M}$ \\
\hline Counter conditioning & $12.90 * *$ & PC, C, PR < A, M \\
\hline Stimulus control & $17.20 * *$ & $\mathrm{PC}, \mathrm{C}, \mathrm{PR}<\mathrm{A}, \mathrm{M}$ \\
\hline
\end{tabular}

Note: $\mathrm{PC}=$ precontemplation; $\mathrm{C}=$ contemplation; $\mathrm{PR}=$ preparation; $\mathrm{A}=$ action; $\mathrm{M}=$ maintenance.

The measure was validated against the stages of change. Consistent with the findings of previous studies [1] [6], the use of each process was at least in precontemplation. Individuals in this stage are not ready to initiate effective stress management, so it is reasonable that they would use the processes less frequently. Differences in the experiential and behavioral processes across the stages showed patterns of increase in the use of these processes, which was largely consistent with the findings of previous studies [1] [6]. The progression through effective stress management stages requires the initiation and maintenance of healthy activities to manage stress. Thus, it is reasonable that the use of both experiential and behavioral processes is more frequent in the action and maintenance stages than in the earlier stages. These results supported the validity of the Korean PPSM version.

The results of this study provide a new measure that can assess the processes of change for effective stress management in Korean college students. Helping college students to manage stress is an important issue for South Korea [4], and this new measure is expected to be used in future studies in South Korea. In addition, the results extend the literature by providing initial evidence that supports the application of the processes of change to stress management in Korean college students. Studies applying processes of change in stress management have been reported from other countries such as United States [2], Japan [6], mainland China [1], and Germany [15], but it had been unknown as to whether this construct would be applicable to the South Korean population. Hence, this extension is important. Nishimura and Chikamoto [9] pointed out the importance of examining the external validity of findings when the findings in some cultures are applied to other cultures. This study predicted the relationships between the stages and processes of change for effective stress management in Korean college students. Therefore, the study results suggested that the processes of change can be applied to effective stress management in Korean college students.

The study has following limitations. First, the participants were recruited at only two colleges. As a result, it remains to be seen how representative the participants were as a sample of Korean college students. This study successfully developed the Korean PPSM version, which can be used for cross-cultural comparative studies with the United States, mainland China, Japan, and South Korea. Based on the study results, it is possible to examine the extent to which the findings can be generalized. Second, it remains unclear whether the Korean PPSM version is appropriate for populations other than students. To assess this, other populations need to be examined based on the study findings.

\section{References}

[1] Deng, K., Tsuda, A., Horiuchi, S. and Matsuda, T. (2013) Relationships between Stages and Processes of Changefor Effective Stress Management in Chinese College Students. Stress Science Research, 28, 74-81. http://dx.doi.org/10.5058/stresskagakukenkyu.28.74

[2] Evers, K.E., Evans, J.L., Fava, J.L. and Prochaska, J.O. (2000) Development and Validation of Trans Theoretical Model Variables Applied to Stress Management. Presented at the 21st Annual Scientific Sessions of the Society of Behavioral Medicine, Nashville. 
[3] Evers, K.E., Prochaska, J.O., Johnson, J. L., Mauriello, L. M., Padula, J. A. and Prochaska, J.M. (2006) A Randomized Clinical Trial of a Population- and Transtheoretical Model-based Stress-management Intervention. Health Psychology, 25, 521-529. http://dx.doi.org/10.1037/0278-6133.25.4.521

[4] Horiuchi, S., Tsuda, A., Kim, E., Hong, K.-S. and Prochaska, J.M. (2010) Relationship between Stage of Change and Self-Efficacy for Stress Management Behavior in Korean University Students. Japanese Journal of Behavioral Medicine, 16, 12-20.

[5] Horiuchi, S., Tsuda, A., Kim, E., Morita, T. and Kobayashi, H. (2013) Effectiveness of the Expert System for Modifying Effective Stress Management Behavior based on the Transtheoretical Model in College Students. Stress Management Research, 9, 69-84.

[6] Horiuchi, S., Tsuda, A., Prochaska, J. M., Kobayashi, H. and Mihara, K. (2012) Relationships between Stages and Processes of Change for Effective Stress Management in Japanese College Students. Psychology, 3, 494-499. http://dx.doi.org/10.4236/psych.2012.36070

[7] Kim, E., Tsuda, A., Horiuchi, S., Park, Y.-S., Kim, U. and Hong, K.-S. (2009) The Relationship between Stages of Change for Stress Management and Depressive Symptoms in a Sample of Korean University Students. Kurume University Psychological Research, 8, 103-110.

[8] Kim, U., Park, Y-S, Kim, E., Tsuda, A. and Horiuchi, S. (2010) The Influence of Parental Social Support and Resiliency of Efficacy on Stress, Depression, and Stress Management Behavior: Comparative Analysis of Elementary School, Middle School and University Students. Korean Journal of Psychology and Social Issues, 16, 197-219.

[9] Nishimura, Y. and Chikamoto, Y. (2005) Health Promotion in Japan: Comparisons with U.S. Perspectives. American Journal of Health Promotion, 19, 213-215. http://dx.doi.org/10.4278/0890-1171-19.3s.213

[10] McEwen, B.S. (2007) Physiology and Neurobiology of Stress and Adaptation: Central Role of the Brain. Physiological Reviews, 87, 873-904. http://dx.doi.org/10.1152/physrev.00041.2006

[11] Pro-Change Behavior Systems, Inc. (2003) Road to Healthy Living: A Guide for Effective Stress Management. Pro-Change Behavior Systems, Inc., Kingston.

[12] Prochaska, J.O. and DiClemente, C.C. (1983) Stages and Processes of Self-Change of Smoking: Toward an Integrative Model of Change. Journal of Consulting and Clinical Psychology, 51, 390-395. http://dx.doi.org/10.1037/0022-006X.51.3.390

[13] ]rochaska, J.O., DiClemente, C.C. and Norcross, J.C. (1992) In Search of How People Change. Applications to Addictive Behaviors, 47, 1102-1114. http://dx.doi.org/10.1037/0003-066X.47.9.1102

[14] Vandenberg, R.J. and Lance, C.E. (2000) A Review and Synthesis of the Measurement Invariance Literature: Suggestions, Practices, and Recommendations for Organizational Research. Organizational Research Methods, 3, 4-70. http://dx.doi.org/10.1177/109442810031002

[15] Vierhausa, M., Maassa, A., Fridricia, M. and Lohausa, A. (2010) Effects of a School-Based Stress Prevention Programme on Adolescents in Different Phases of Behavioural Change. Educational Psychology, 30, 465-480. http://dx.doi.org/10.1080/01443411003724624 